\title{
Entanglement harvesting in double-layer graphene by vacuum fluctuations in a microcavity
}

\author{
Juan Sebastián Ardenghi* \\ Departamento de Física, Universidad Nacional del Sur, \\ Avenida Alem 1253, B8000CPB Bahía Blanca, Argentina \\ and Instituto de Física del Sur (IFISUR, UNS-CONICET), \\ Avenida Alem 1253, B8000CPB Bahía Blanca, Argentina
}

(Received 27 June 2018; published 8 August 2018)

\begin{abstract}
The aim of this work is to study the entanglement harvesting between two graphene layers inside a planar microcavity. Applying time-dependent perturbation theory it is shown that nonclassical correlations between electrons in different layers are obtained through the exchange of virtual photons. Considering different initial states of the electrons and the vacuum state of the electromagnetic field, the negativity measure that quantifies the entanglement is computed through the photon propagator for time scales smaller than the light-crossing time of the double layer. The results are compared with those obtained for hydrogenic probes and pointlike Unruh-DeWitt detectors, showing that for different initial states, entangled $X$ states and more general entangled reduced matrices are obtained, which enlarge the classification of bipartite quantum states.
\end{abstract}

DOI: 10.1103/PhysRevD.98.045006

\section{INTRODUCTION}

The vacuum state of a free quantum field contains correlations of different observables in separate regions of spacetime, even when those regions are spacelike separated [1-3]. This nonclassical behavior of the vacuum state of the field is a vital concept in phenomena such as quantum collect calling [4], the black hole information loss problem [5,6], and quantum energy teleportation [7-9]. These correlations are, in principle, physically accessible because they can be obtained from the field vacuum via quantum particle detectors that couple to it locally [10]. This allows to observe an entanglement of the particle detectors that are operated by observers, even if they remain spacelike separated during their whole existence [2]. The phenomenon of extraction of nonclassical correlations from the quantum vacuum has become known as entanglement harvesting, which was introduced in Ref. [1]. Entanglement harvesting from scalar fields has been widely studied [3] and applied in entanglement farming [11], metrology [12], and in cosmology, where it has been shown that entanglement harvesting is very sensitive to the geometry of the underlying spacetime [13,14] or even its topology [15]. In general, the detector-field interaction is modeled using the Unruh-DeWitt model [16], which consists of a linear coupling of a pointlike two-level quantum system and a massless (or not) scalar quantum field, where a spatial smearing function is included in order to allow the

*jsardenghi@gmail.com two-level system to have a finite extension in space. ${ }^{1}$ Experimental implementations of the Unruh-DeWitt model have been developed in atomic systems and superconducting circuits [17-19], where in the former an alkali atom as a first quantized system can serve as a detector for the second quantized electromagnetic field.

Nevertheless, and despite its great success, the UnruhDeWitt model cannot capture the complete interaction between atoms and the electromagnetic field vacuum. The electromagnetic field is a vector field and carries angular momentum, but this implies that any study based on the Unruh-DeWitt model will not capture the anisotropies and orientation dependence of the entanglement harvesting and will not predict any effect related to the exchange of the angular momentum of the atoms with the quantum field. In Ref. [20], a dipole coupling between the electromagnetic field and hydrogenoid atoms was studied exhaustively. In turn, particle detector models are ubiquitous as models for experimental setups in quantum optics [21]. The most usual light-matter interaction models - the Jaynes-Cummings model and its variantsare almost identical to the Unruh-DeWitt model [21], where the rotating wave approximation is applied, and the terms proportional to $\sigma^{+} a^{\dagger}$ and the Hermitian conjugate are removed from the Hamiltonian. The reason behind this approximation is that the neglected terms yield bounded oscillations when integrated in time for the detector-field

\footnotetext{
${ }^{1}$ In turn, a time-window function is included in the interaction to allows the interaction to occur in a finite time.
} 
resonance. These bounded oscillations can be neglected in the detector-field dynamics compared to the close-toresonance rotating wave terms. Removing these terms implies that microcausality is not guaranteed and that the Hamiltonian is no longer linear in the field [22].

On the other hand, graphene - a monolayer of carbon atoms-has garnered considerable interest because it is attractive for various electronic and magnetic applications [23-25]. Besides its novel high-speed electronics properties [26], graphene is of great interest from the point of view of fundamental physics as well. The low-energy electron excitations in graphene are massless Dirac fermions with a linear energy spectrum [27,28]. This makes graphene a condensed-matter playground to study various relativistic quantum phenomena, such as Klein tunneling and the Casimir effect $[29,30]$. Up to now, most graphene-related studies focused on its unusual transport properties, but quantum effects arising from interactions with a quantized electromagnetic field have been neglected.

Recently, quantum electromagnetic field effects have been studied with the purpose of opening a band gap in the spectrum by illuminating graphene with circularly polarized light [31]. In this case, the gap appears due to the formation of composite electron-photon states which are similar to polaritons in ionic crystals and quantum microcavities $[32,33]$. It should be noted that within the framework of QED, the excitonic effects can be observed even if real photons are absent and electrons interact only with vacuum fluctuations of the electromagnetic field by emitting and reabsorbing virtual photons [34]. From this, it would be natural to expect that the photon-induced splitting of the valence and conductivity bands in graphene [31] will arise due to the vacuum fluctuations even in the absence of external field pumping. These effects can be observed by decreasing the effective volume where electron-photon interactions take place, which can be accomplished by embedding an electron system inside a planar microcavity [33].

When the electromagnetic field is coupled to graphene in the long-wavelength approximation, the minimal coupling $\mathbf{p} \rightarrow \mathbf{p}-e \mathbf{A}$ must be applied to the Hamiltonian, which naturally introduces the Unruh-DeWitt interaction between the detector (in this case, the sublattice basis) and the quantum field. This allows to study the entanglement harvesting between two graphene sheets inside a planar microcavity and, in particular, to study the photon-induced splitting of the valence and conduction bands at small times. From the conceptual viewpoint, this is a generalization of entanglement harvesting to extended or surface systems and not pointlike systems, such as atomic probes or two-level systems. It should be stressed that separated electron systems (such as double-layer graphene) remain strongly coupled by electron-electron interactions even when they cannot exchange particles, provided that the layer separation $d$ is comparable to a characteristic distance $l$ between charge carriers within layers [35]. One of the consequences of this remote coupling is a phenomenon called Coulomb drag, in which an electric current passing through one of the layers causes frictional charge flow in the other layer and reveals many unpredicted features in double-layer graphene, such as a larger Coulomb drag when both layers are neutral [36]. Although this phenomenon is considerable for double-layer graphene in a cavity, when entanglement harvesting-due to the vacuum fluctuations of the electromagnetic field cavity being studiedtime scales much smaller than the light-crossing time between the layers are considered and the Coulomb drag can be neglected, or (from the point of view of quantum field theory) we can consider the Coulomb interaction between the layers in the spacetime region where causality is violated.

Thus, in this work we study entanglement harvesting between two graphene sheets inside a cavity, where the monopole detector is given by the natural interaction of the electrons in graphene with the electromagnetic field. In particular, the raising and lowering operators that act as the detector are obtained through the Pauli matrices, which act on the sublattice basis. When the initial states of electrons are written as eigenstates of the free Hamiltonian, the effect of the interaction is not trivial because these eigenstates are written as superpositions of the sublattice basis. In turn, when the initial states of the electrons are given in a defined sublattice basis, the entanglement harvesting obtained is identical to that obtained in Ref. [10], with the main difference coming from the smearing of the detectors, which in this work are represented by the graphene sheets.

This paper is organized as follows. In Sec. II we introduce the formalism to compute the time-dependent perturbation theory. In Sec. III, we present our results and discussions for different initial states of electrons in both graphene sheets, and make a comparison with the UnruhDeWitt detector. In Sec. IV we present our conclusions. In Appendices A and B we present a detailed calculation of the photon propagator and the second-order contribution to the reduced quantum operator in time-dependent perturbation theory.

\section{THE MODEL}

The Hamiltonian of the double-layer graphene coupled to the electromagnetic field of the cavity reads (see Fig. 1)

$$
H=\sum_{i=1,2}\left(v_{F} \sigma_{i} \mathbf{p}_{i}-e v_{F} \sigma_{i} \mathbf{A}_{i}\right)+H_{F},
$$

where $i$ runs over the two electrons, each in different graphene layers, ${ }^{2}$ which can be in either the valence or conduction band, $\mathbf{A}_{i}$ is the potential vector acting on

\footnotetext{
${ }^{2}$ The index $i$ should not be confused with the index notation of a vector.
} 


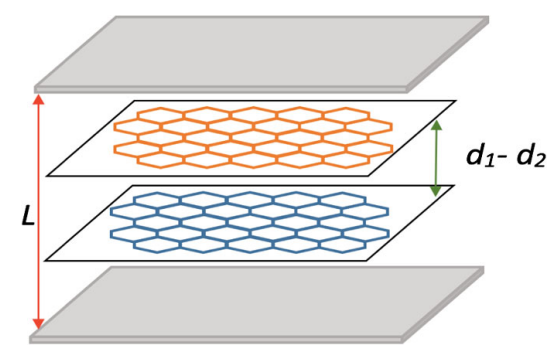

FIG. 1. The device setup of double-layer graphene inside a planar microcavity.

each electron, and $H_{F}$ is the Hamiltonian of the electromagnetic field $H_{F}=\sum_{n, \mathbf{q}, \lambda} \hbar \omega_{n, \mathbf{q}, \lambda} a_{n \mathbf{q} \lambda}^{\dagger} a_{n \mathbf{q} \lambda}$, where $\omega_{n, \mathbf{q}}=$ $c \sqrt{q^{2}+\left(\frac{\pi n}{L}\right)^{2}}$ and $a_{n \mathbf{q} \lambda}\left(a_{n \mathbf{q} \lambda}^{\dagger}\right)$ are the creation and annihilation operators of the cavity field that obeys the usual commutation relations $\left[a_{n \mathbf{q} \lambda}, a_{n^{\prime} \mathbf{q}^{\prime} \lambda^{\prime}}^{\dagger}\right]=\delta_{n, n^{\prime}} \delta_{\lambda, \lambda^{\prime}} \delta_{\mathbf{q q} \mathbf{q}^{\prime}} I$ [31]. The quantum electromagnetic field can be written in terms of the creation and annihilation operators for each mode $\mathbf{q}$ with frequency $\omega_{n, \mathbf{q}}$ and polarization $\lambda$ as [37]

$$
\begin{aligned}
\mathbf{A}_{i}(\mathbf{r}, z, t)= & \sum_{\lambda= \pm ; \mathbf{n}, \mathbf{q}} \frac{\gamma}{\sqrt{\omega_{\mathbf{n}, \mathbf{q}}}} \sin \left(\frac{\pi n d_{i}}{L}\right)\left(\hat{e}_{\mathbf{q} \lambda} a_{n \mathbf{q} \lambda} e^{i\left(\mathbf{q} \cdot \mathbf{r}-\omega_{\mathbf{n}, \mathbf{q}} \mathbf{t}\right)}\right. \\
& \left.+\hat{e}_{\mathbf{q} \lambda} a_{n \mathbf{q} \lambda}^{\dagger} e^{-i\left(\mathbf{q} \cdot \mathbf{r}-\omega_{\mathbf{n}, \mathbf{q}} \mathbf{t}\right)}\right)
\end{aligned}
$$

where $\hat{e}_{\mathbf{q} \lambda}$ are the polarization directions orthogonal to the in-plane wave vector of the field $\mathbf{q}, L$ is the distance between the two mirrors of the planar cavity, $S$ is the area of the graphene sample $\gamma=\sqrt{\frac{\hbar}{\epsilon L S}}$, and $n$ is the mode index in the $z$ direction. By considering one layer of graphene placed at $z=d_{1}$ and the second one at $z=d_{2}$, the potential vectors $A_{i}$ are given by Eq. (2) with the replacements $z=d_{1}$ and $z=d_{2}$, respectively. By considering $H_{0}=$ $\sum_{i=1,2} v_{F} \sigma_{i} \mathbf{p}_{i}$ (the unperturbed Hamiltonian), a set of eigenstates can be obtained in terms of the sublattice basis,

$$
\left|\mathbf{k}_{i}, s_{i}\right\rangle=\frac{e^{i \mathbf{k}_{i} \cdot \mathbf{r}}}{\sqrt{2 S}}\left(\left|A_{i}\right\rangle+s e^{i \theta_{i}}\left|B_{i}\right\rangle\right),
$$

where $\theta_{i}=\arctan \left(\frac{k_{y_{i}}}{k_{x_{i}}}\right)$ is the angle of the wave vector with respect the $x$ axis, and $s= \pm 1$ for the conduction and valence bands. ${ }^{3}$ The elementary electromagnetic field excitations from the vacuum can be characterized by the wave vector $\mathbf{q}$ and the helicity, which can be constructed through the polarization vectors $\hat{e}_{x}$ and $\hat{e}_{y}$ by redefining $\hat{e}_{+}=\frac{1}{\sqrt{2}}\left(\hat{e}_{x}+i \hat{e}_{y}\right)$ and $\hat{e}_{-}=\frac{1}{\sqrt{2}}\left(\hat{e}_{x}-i \hat{e}_{y}\right)$. In order to

\footnotetext{
${ }^{3}$ In the low-wavelength approximation, the wave vector can be approximated at one of the two inequivalent symmetry points of the Brillouin zone - the $K$ or $K^{\prime}$ valleys. For the sake of simplicity we will consider one valley.
}

express the dot product $\sigma_{i} \mathbf{A}_{i}$ we have to consider a twodimensional space orthogonal to the $z$ direction. By using the circular polarization basis, the dot product reads ${ }^{4}$

$$
\sigma_{i} \mathbf{A}_{i}=\left(\sigma_{x}^{(i)} \hat{e}_{x}+\sigma_{y}^{(i)} \hat{e}_{y}\right)\left(A_{i}^{+} \hat{e}_{+}+A_{i}^{-} \hat{e}_{-}\right)=\sqrt{2} \sum_{\lambda= \pm} \sigma_{-\lambda}^{(i)} A_{\lambda}^{(i)}
$$

where $\lambda= \pm 1$ for both helicities, $\sigma_{\lambda}^{(i)}=\frac{1}{2}\left(\sigma_{x}^{(i)}+\lambda i \sigma_{y}^{(i)}\right)$, and where

$A_{\lambda}^{(i)}=\sum_{n, \mathbf{q}} \frac{\gamma \sin \left(\frac{\pi n d_{i}}{L}\right)}{\sqrt{\omega_{n, \mathbf{q}}}}\left(a_{n \mathbf{q} \lambda} e^{i\left(\mathbf{q} \cdot \mathbf{r}-\omega_{\mathbf{n}, \mathbf{q}} \mathbf{t}\right)}+a_{n \mathbf{q} \lambda}^{\dagger} e^{-i\left(\mathbf{q} \cdot \mathbf{r}-\omega_{\mathbf{n}, \mathbf{q}} \mathbf{t}\right)}\right)$.

In order to compute the coupling between the valence and conduction bands with the circular polarized photons, the following relations must be taken into account: $\sigma_{+}|\mathbf{k}, A\rangle=0, \quad \sigma_{+}|\mathbf{k}, B\rangle=|\mathbf{k}, A\rangle, \quad \sigma_{-}|\mathbf{k}, A\rangle=|\mathbf{k}, B\rangle$, $\sigma_{-}|\mathbf{k}, B\rangle=0, \sigma_{+}|\mathbf{k},+\rangle=\frac{1}{2} e^{i \eta_{\mathbf{k}}}(|\mathbf{k},+\rangle+|\mathbf{k},-\rangle), \sigma_{-}|\mathbf{k},+\rangle=$ $\frac{1}{2} e^{-i \eta_{\mathbf{k}}}(|\mathbf{k},+\rangle-|\mathbf{k},-\rangle), \sigma_{+}|\mathbf{k},-\rangle=-\frac{1}{2} e^{i \eta_{\mathbf{k}}}(|\mathbf{k},+\rangle+|\mathbf{k},-\rangle)$, and $\sigma_{-}|\mathbf{k},-\rangle=\frac{1}{2} e^{-i \eta_{\mathbf{k}}}(|\mathbf{k},+\rangle-|\mathbf{k},-\rangle$ ) (see Ref. [31]). From these relations we can see that this model is similar to those used in entanglement harvesting from two detectors [20], where $\sigma_{ \pm}$are the detector's energy raising and lowering operators. In this work, this two-level system is the sublattice basis, which implies that one photon with a definitive helicity is absorbed whenever a delocalized electron jumps from the $A$ sublattice to the $B$ sublattice, or a photon is emitted when an electron jumps from the $B$ sublattice to the $A$ sublattice. But the stationary states in graphene are given by the eigenvectors of the Hamiltonian which can be written as superpositions in the sublattice basis [see Eq. (3)]. This implies that the entanglement harvesting of the two graphene layers is more subtle because the absorbtion and emission of virtual photons imply a superposition of valence and conduction bands with definite incoming and outgoing momenta. In turn, the system under study is a generalization of pointlike systems, where the monopole detectors raise and lower the two discrete energy levels. In the case of double-layer graphene, the detector is given by the interaction $\sigma \mathbf{A}$, where $\mathbf{A}$ is now evaluated in each graphene layer. The entanglement harvesting on surfaces implies at least two energy bands, and the possible transitions are ruled by the energy conservation given by the momentum of the electrons in both graphene sheets. In the literature, entanglement harvesting is investigated using a pointlike approximation for the detector model, which has no extension and interacts with the field only at the spacetime point where it is placed. This

\footnotetext{
${ }^{4} \mathrm{We}$ are assuming that the virtual photons interacting with the graphene layers propagate normally with respect to these layers.
} 
assumption, which can be considered an approximation for real detectors with finite size, results in ultraviolet divergences. Several regularization schemes yield different transition probabilities [38]. In the case of double-layer graphene, this problem is not present due to the natural spatial smearing of the interaction between the electromagnetic field and graphene sheets.

In order to compute the entanglement of electrons we can work perturbatively to second order in the interacting Hamiltonian $H_{\text {int }}=\sqrt{2} \sum_{\lambda= \pm} \sigma_{-\lambda}^{(i)} A_{\lambda}^{(i)}$, where the interaction picture time evolution operator $U$ for the full system is

$$
U=U^{(0)}+U^{(1)}+U^{(2)}+\cdots,
$$

where $U^{(0)}=I, U^{(1)}=-i \int_{-\infty}^{t} d t^{\prime} H_{\text {int }}\left(t^{\prime}\right), U^{(2)}=-\int_{-\infty}^{t} d t^{\prime} \times$ $H_{\text {int }}\left(t^{\prime}\right) \int_{-\infty}^{t^{\prime}} d t^{\prime \prime} H_{\text {int }}\left(t^{\prime \prime}\right)$, and $H_{\text {int }}(t)=e^{-i\left(H_{0}+H_{F}\right) t} V(t) \times$ $e^{i\left(H_{0}+H_{F}\right) t}$. Then, given an initial density matrix $\rho_{0}$, the final density matrix $\rho_{T}$ is hence given by

$$
\begin{aligned}
\rho_{T} & =U \rho_{0} U^{\dagger} \\
& =\left[I+U^{(1)}+U^{(2)}+\cdots\right] \rho_{0}\left[I+U^{(1)}+U^{(2)}+\cdots\right] .
\end{aligned}
$$

If we write $\rho_{T}=\rho_{0}+\rho_{T}^{(1)}+\rho_{T}^{(2)}+\ldots$, then

$$
\begin{aligned}
& \rho_{T}^{(1)}=U^{(1)} \rho_{0}+\rho_{0} U^{(1) \dagger}, \\
& \rho_{T}^{(2)}=U^{(1)} \rho_{0} U^{(1) \dagger}+U^{(2)} \rho_{0}+\rho_{0} U^{(2) \dagger} .
\end{aligned}
$$

In order to rearrange the notation, we can write $\rho_{T}^{(i, j)}=$ $U^{(i)} \rho_{0} U^{(j) \dagger}$, and therefore the time-evolved density matrix can be written as a sum of terms of the form $\rho=$ $\rho_{0}+\rho^{(1,0)}+\rho^{(0,1)}+\rho^{(2,0)}+\rho^{(0,2)}+\rho^{(1,1)}+\cdots$ Because we are going to analyze entanglement and correlation harvesting of both graphene layers from the vacuum fluctuations of the quantum electromagnetic field, we can consider that the initial state of the electron-electron quantum field system is

$$
\rho_{0}=\left|\Omega_{0}\right\rangle\left\langle\Omega_{0}\right| \otimes \rho_{G},
$$

where $\left|\Omega_{0}\right\rangle=\left|\Omega_{0}^{(+)}, \Omega_{0}^{(-)}\right\rangle$is the vacuum state of the electromagnetic field with circular polarization \pm and $\rho_{G}$ is the initial density matrix of the electron-electron system, where without loss of generality we can consider that both electrons are in the conduction band with momenta $\mathbf{k}_{\mathbf{1}}$ and $\mathbf{k}_{\mathbf{2}}$, respectively, or both electrons are in the sublattice basis $A$ with momenta $\mathbf{k}_{\mathbf{1}}$ and $\mathbf{k}_{\mathbf{2}}$, respectively. We are interested in the partial state of the electrons in the graphene sheet after the interaction with the quantum field, which is given by

$$
\rho(t)=\operatorname{Tr}_{A}\left(U \rho_{0} U^{\dagger}\right) .
$$

This means that the nondiagonal terms in the field produced by time evolution will not be relevant for our purposes. In particular, any contribution for which the parities of $i$ and $j$ are different will give a zero contribution to the electrons in graphene final states as long as the initial state of the field is diagonal in the Fock basis, which is the case for the vacuum or any incoherent superposition of Fock states such as a thermal state. Then, the unique term to be computed is $\rho_{T}^{(2)}$ and the trace over the field basis must be carried out. The $\operatorname{Tr}_{A}\left(U^{(2)} \rho_{0}\right)=-\frac{1}{2} \int_{-\infty}^{t} \int_{-\infty}^{t} d t_{1} d t_{2} \operatorname{Tr}_{A}\left[H_{\text {int }}\left(t_{1}\right) H_{\text {int }}\left(t_{2}\right) \rho_{0}\right]$ contribution in Eq. (8) can be written as

$$
\operatorname{Tr}_{\phi}\left(U^{(2)} \rho_{0}\right)=-\left(e v_{F}\right)^{2} \sum_{i, j=1,2 ; \lambda, \lambda^{\prime}} \int d^{2} \mathbf{r}_{1} \int d^{2} \mathbf{r}_{2} \int_{-\infty}^{t} \int_{-\infty}^{t} d t_{1} d t_{2} \Delta_{\lambda, \lambda^{\prime}}^{(i, j)}\left(\mathbf{r}_{1}, t_{1}, \mathbf{r}_{2}, t_{2}\right) \sigma_{-\lambda}^{(i)}\left(t_{1}\right) \sigma_{-\lambda^{\prime}}^{(j)}\left(t_{2}\right) \rho_{G}
$$

where

$$
\Delta_{\lambda, \lambda^{\prime}}^{(i, j)}\left(\mathbf{r}_{1}, t_{1}, \mathbf{r}_{2}, t_{2}\right)=\left\langle\Omega_{0}\left|A_{\lambda}^{(i)}\left(t_{1}\right) A_{\lambda^{\prime}}^{(j)}\left(t_{2}\right)\right| \Omega_{0}\right\rangle=\delta_{\lambda \lambda^{\prime}} \sum_{n, \mathbf{q}} \frac{\gamma^{2}}{\omega_{n, \mathbf{q}}} \sin \left(\frac{\pi n d_{i}}{L}\right) \sin \left(\frac{\pi n d_{j}}{L}\right) e^{i\left(\mathbf{q} \cdot \mathbf{r}_{1}-\omega_{\mathbf{n}, \mathbf{q}} \mathbf{t}_{1}\right)} e^{-i\left(\mathbf{q} \cdot \mathbf{r}_{2}-\omega_{\mathbf{n}, \mathbf{q}} \mathbf{t}_{2}\right)}
$$

is the photon propagator, where we have used that $A_{\lambda}^{(i)}(\mathbf{r}, t)=e^{\frac{i}{\hbar} H_{0 F} t} A_{\lambda}^{(i)} e^{-\frac{i}{\hbar} H_{0 F} t}$ and where $\sigma_{-\lambda}^{(i)}(t)=e^{\frac{i}{\hbar} H_{0 S} t} \sigma_{-\lambda}^{(i)} e^{-\frac{i}{\hbar} H_{0 S} t}$. The photon propagator can be computed exactly (see Appendix A) and the result reads $\Delta_{\lambda, \lambda^{\prime}}^{(i, j)}(\Delta t,|\Delta \mathbf{r}|)=\delta_{\lambda \lambda^{\prime}} F_{i j}(|x|)$, where

$$
F_{i j}(|x|)=\frac{\gamma^{2} \sin \left(\frac{\pi d_{i}}{L}\right) \sin \left(\frac{\pi d_{j}}{L}\right) \sinh \left(\frac{\pi|x|}{L}\right)}{16 \pi|x| \sin \left(\frac{\pi\left(d_{i}-d_{j}-i|x|\right)}{2 L}\right) \sin \left(\frac{\pi\left(d_{i}+d_{j}-i|x|\right)}{2 L}\right) \sin \left(\frac{\pi\left(d_{i}-d_{j}+i|x|\right)}{2 L}\right) \sin \left(\frac{\pi\left(d_{i}+d_{j}+i|x|\right)}{2 L}\right)},
$$

where $|x|=\sqrt{c^{2} \Delta t^{2}-|\Delta \mathbf{r}|^{2}}$, with $\Delta t=t_{1}-t_{2}$ and $\Delta \mathbf{r}=$ $\mathbf{r}_{1}-\mathbf{r}_{2}$. In the last equation, the infinite sum of modes has been carried out, although it is known that realistic cavities are not good cavities for the whole frequency spectrum; thus, an improved version of the model introduced in this work should introduce a mode cutoff. Nevertheless, this cutoff would imply that the usual light-matter interaction violates causality. Then, although the model is ideal and does not represent real cavities, it is consistent with causality. In a similar way, the other two contributions to $\rho$ at second order read 


$$
\operatorname{Tr}_{\phi}\left(\rho_{0} U^{(2) \dagger}\right)=-\left(e v_{F}\right)^{2} \sum_{i, j=1,2 ; \lambda, \lambda^{\prime}} \int d^{2} \mathbf{r}_{1} \int d^{2} \mathbf{r}_{2} \int_{-\infty}^{t} \int_{-\infty}^{t} d t_{1} d t_{2} \Delta_{\lambda, \lambda^{\prime}}^{(i j)}\left(\mathbf{r}_{1}, t_{1}, \mathbf{r}_{2}, t_{2}\right) \rho_{G} \sigma_{-\lambda}^{(i) \dagger}\left(t_{1}\right) \sigma_{-\lambda^{\prime}}^{(j) \dagger}\left(t_{2}\right)
$$

and

$$
\operatorname{Tr}_{\phi}\left(U^{(1)} \rho_{0} U^{(1) \dagger}\right)=2\left(e v_{F}\right)^{2} \sum_{i, j=1,2 ; \lambda, \lambda^{\prime}} \int_{-\infty}^{t} \int_{-\infty}^{t} d t_{1} d t_{2} \Delta_{\lambda, \lambda^{\prime}}^{(i, j) *}\left(\mathbf{r}_{1}, t_{1}, \mathbf{r}_{2}, t_{2}\right) \sigma_{-\lambda}^{(i)}\left(t_{1}\right) \rho_{G} \sigma_{-\lambda}^{(j) \dagger}\left(t_{2}\right) .
$$

Collecting all of the terms, the reduced state reads

$$
\begin{aligned}
\rho=\operatorname{Tr}_{\phi}(\rho(t))= & -\left(e v_{F}\right)^{2} \sum_{i, j=1,2 ; \lambda} \int_{-\infty}^{t} \int_{-\infty}^{t} d t_{1} d t_{2} \Delta_{\lambda, \lambda^{\prime}}^{(i j)}\left(\mathbf{r}_{1}, t_{1}, \mathbf{r}_{2}, t_{2}\right) \\
& \times\left[\sigma_{-\lambda}^{(i)}\left(t_{1}\right) \sigma_{-\lambda}^{(j)}\left(t_{2}\right) \rho_{G}+\rho_{G} \sigma_{-\lambda}^{(i) \dagger}\left(t_{1}\right) \sigma_{-\lambda}^{(j) \dagger}\left(t_{2}\right)-2 \sigma_{-\lambda}^{(i)}\left(t_{1}\right) \rho_{G} \sigma_{-\lambda}^{(j) \dagger}\left(t_{2}\right)\right] .
\end{aligned}
$$

In Fig. 2 the photon propagator in the cavity is shown as a function of $|x|$ for $d_{1} / L=0.4$ and $d_{2} / L=0.6$. As it can be seen, the propagator does not vanish outside the light cone, which implies the emergence of correlations between the two graphene sheets at $t<c /\left|d_{2}-d_{1}\right|$. This implies the generation of a correlated state from an uncorrelated one only by local interactions because the field vacuum is an entangled state between spacelike separated regions. In turn, the nonzero probability of an electron in the graphene sheet to get excited outside the light cone is independent of the remaining electron in the other graphene sheet, and thus no information is carried over a spacelike distance. The main difference between the result obtained for $\rho$ in double-layer graphene and the pointlike detectors is the spatial integration over the constrained space in which the electrons can move. When real detectors are modeled, a smeared function must be introduced in the interaction which introduces the spatial integration (see Ref. [39]). Both electrons are delocalized in each graphene sheet and can become entangled by merely letting them interact with the field vacuum state. The system becomes entangled because they swap entanglement from the vacuum rather than by interacting through the exchange of real field quanta.

Finally, the matrix elements $\left\langle\mathbf{k}_{1}^{\prime}, s_{1}^{\prime}, \mathbf{k}_{2}^{\prime}, s_{2}^{\prime}\left|\operatorname{Tr}_{\phi}\left(U^{(2)} \rho_{0}\right)\right|\right.$ $\left.\mathbf{k}_{1}, s_{1}, \mathbf{k}_{2}, s_{2}\right\rangle$ read (see Appendix B)

$$
\begin{aligned}
\left\langle\mathbf{k}_{1}^{\prime}, s_{1}^{\prime}, \mathbf{k}_{2}^{\prime}, s_{2}^{\prime}|\rho(t)| \mathbf{k}_{1}, s_{1}, \mathbf{k}_{2}, s_{2}\right\rangle= & -\left(e v_{F}\right)^{2} \delta\left(\mathbf{k}_{\mathbf{1}}-\mathbf{k}_{\mathbf{1}}^{\prime}+\mathbf{k}_{\mathbf{2}}-\mathbf{k}_{\mathbf{2}}^{\prime}\right) \sum_{i, j=1,2 ; \lambda} \int_{0}^{t} \int_{0}^{t} d t_{1} d t_{2} \mathcal{F}_{i j}\left(\mathbf{k}_{\mathbf{2}}-\mathbf{k}_{\mathbf{2}}^{\prime}, \Delta t\right) \\
& \times\left\langle s_{1}^{\prime}, s_{2}^{\prime}\left|\left(\sigma_{-\lambda}^{(i)}\left(t_{1}\right) \sigma_{-\lambda}^{(j)}\left(t_{2}\right) \rho_{G}-2 \sigma_{-\lambda}^{(i)}\left(t_{1}\right) \rho_{G} \sigma_{-\lambda}^{(j) \dagger}\left(t_{2}\right)+\rho_{G} \sigma_{-\lambda}^{(i) \dagger}\left(t_{1}\right) \sigma_{-\lambda}^{(j) \dagger}\left(t_{2}\right)\right)\right| s_{1}, s_{2}\right\rangle,
\end{aligned}
$$

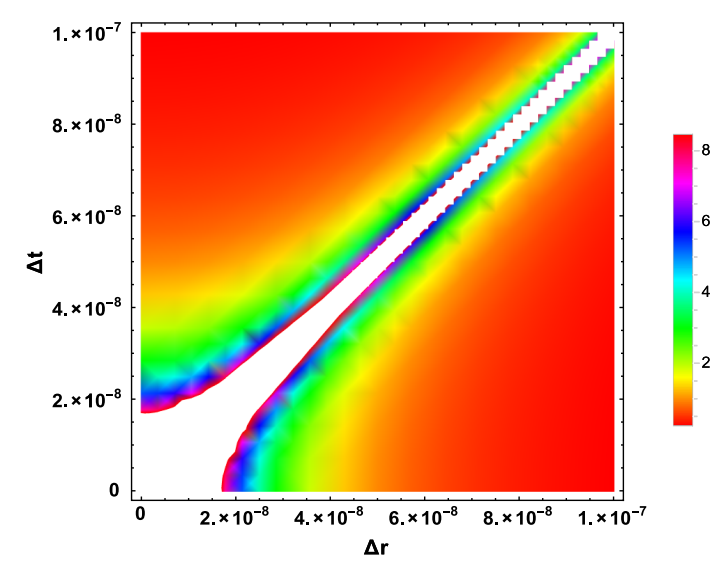

FIG. 2. Photon cavity propagator as a function of space and time. where [see Eq. (B7)]

$$
\begin{aligned}
\mathcal{F}_{i j} & \left(\mathbf{k}_{\mathbf{2}}-\mathbf{k}_{\mathbf{2}}^{\prime}, \Delta t\right) \\
= & \int d^{2} \Delta \mathbf{r} e^{-i\left(\mathbf{k}_{\mathbf{2}}-\mathbf{k}_{2}^{\prime}\right) \cdot \Delta \mathbf{r}} F_{i j}\left(\sqrt{\Delta t^{2}-|\Delta \mathbf{r}|^{2}}\right)
\end{aligned}
$$

and $\left|s_{1}, s_{2}\right\rangle=\left|s_{1}\right\rangle \otimes\left|s_{2}\right\rangle$ is an arbitrary basis, e.g., the sublattice basis (in which case $s_{i}=A, B$ ) or the valenceconduction band basis (in which case $s_{i}= \pm$ ). The Dirac delta $\delta\left(\mathbf{k}_{\mathbf{1}}-\mathbf{k}_{\mathbf{1}}^{\prime}+\mathbf{k}_{\mathbf{2}}-\mathbf{k}_{\mathbf{2}}^{\prime}\right)$ implies momentum conservation and $\mathbf{k}_{\mathbf{i}}\left(\mathbf{k}_{\mathbf{i}}^{\prime}\right)$ is the initial (final) momentum of both electrons.

\section{RESULTS AND DISCUSSIONS}

In order to obtain the critical parameters in which the reduced quantum state is entangled, we can expand $\operatorname{Tr}_{\phi}(\rho(t))$ in small values of $t$ in Eq. (16), 


$$
\begin{aligned}
\left\langle\mathbf{k}_{1}^{\prime}, s_{1}^{\prime}, \mathbf{k}_{2}^{\prime}, s_{2}^{\prime}|\rho(t)| \mathbf{k}_{1}, s_{1}, \mathbf{k}_{2}, s_{2}\right\rangle= & -\left(e v_{F} t\right)^{2} \delta\left(\mathbf{k}_{\mathbf{1}}-\mathbf{k}_{\mathbf{1}}^{\prime}+\mathbf{k}_{\mathbf{2}}-\mathbf{k}_{\mathbf{2}}^{\prime}\right) \\
& \times \sum_{i, j=1,2 ; \lambda} \mathcal{F}_{i j}\left(\mathbf{k}_{\mathbf{2}}-\mathbf{k}_{\mathbf{2}}^{\prime}, 0\right) \times\left\langle s_{1}^{\prime}, s_{2}^{\prime}\left|\left(\sigma_{-\lambda}^{(i)} \sigma_{-\lambda}^{(j)} \rho_{G}-2 \sigma_{-\lambda}^{(i)} \rho_{G} \sigma_{\lambda}^{(j)}+\rho_{G} \sigma_{\lambda}^{(i)} \sigma_{\lambda}^{(j)}\right)\right| s_{1}, s_{2}\right\rangle,
\end{aligned}
$$

where we have used that $\sigma_{-\lambda}^{(i) \dagger}=\sigma_{\lambda}^{(i)}$. Considering as initial state $\rho_{G}=|A, A\rangle\langle A, A|$ where both electrons in each graphene sheet have nonzero amplitude in the $A$ sublattice basis, the normalized reduced quantum state can be written in the basis $|A, A\rangle,|A, B\rangle,|B, A\rangle$, and $|B, B\rangle$ as

$$
\rho(t)=\left[\begin{array}{cccc}
1-2\left(e v_{F}\right)^{2} t^{2}\left[\mathcal{F}_{11}+\mathcal{F}_{22}\right] & 0 & 0 & 2\left(e v_{F}\right)^{2} t^{2} \mathcal{F}_{12} \\
0 & 2\left(e v_{F}\right)^{2} t^{2} \mathcal{F}_{22} & -2\left(e v_{F}\right)^{2} t^{2} \mathcal{F}_{12} & 0 \\
0 & -2\left(e v_{F}\right)^{2} t^{2} \mathcal{F}_{12} & 2\left(e v_{F}\right)^{2} t^{2} \mathcal{F}_{11} & 0 \\
2\left(e v_{F}\right)^{2} t^{2} \mathcal{F}_{12} & 0 & 0 & 0
\end{array}\right],
$$

where $\mathcal{F}_{i j}$ is a function of $\mathbf{k}_{\mathbf{2}}-\mathbf{k}_{\mathbf{2}}^{\prime}$ and momentum conservation is understood. This density matrix has the form of the so-called $X$ state [40] and is positive at leading order in $O\left(\left(\gamma e v_{F}\right)^{2}\right)$ and all the perturbative corrections of $\rho$ to the final density matrix are traceless. Therefore, the trace of the final state of $\rho$ is always preserved, independent of up to which order $O(n)$ in the coupling constant the corrections are taken into account.

The $X$ states are those in which several matrix elements are zero $\left(\rho_{12}=\rho_{13}=\rho_{24}=\rho_{34}=0\right)$ [41]. In turn, many well-known and useful families of states have an $X$ form, including the Bell states, Werner states [42], and isotropic states [41]. Recently, it was shown numerically that all twoqubit mixed states are equivalent to $X$ states by a single entanglement-preserving unitary transformation, so concurrence and other entanglement measures of such an $X$ state are equal to those of the original general state [43]. In

general, a density matrix is said to be inseparable or entangled if it cannot be expressed as a convex sum of local density matrices [42]. In the present case of a $2 \times 2$ system, a necessary and sufficient condition for inseparability is that the negativity be positive, where the negativity $\mathcal{N}$ is defined as the lowest eigenvalue of the partial transpose of $\rho$ [44-46]. The negativity is an entanglement monotone that for two-qubit settings only vanishes for separable states and is defined as

$$
\mathcal{N}(\rho)=\sum_{\alpha \in \sigma\left[\rho^{\Gamma_{2}}\right]} \frac{\left|\alpha_{i}\right|-\alpha_{i}}{2},
$$

where $\alpha_{i}$ are the eigenvalues of the partial transpose of $\rho^{\Gamma_{2}}=(I \otimes T) \rho$ with respect to the second system. This partial transpose reads

$$
\rho^{\Gamma_{2}}=(I \otimes T) \rho=\left[\begin{array}{cccc}
1-2\left(e v_{F} t\right)^{2}\left[\mathcal{F}_{11}+\mathcal{F}_{22}\right] & 0 & 0 & -2\left(e v_{F} t\right)^{2} \mathcal{F}_{12} \\
0 & 2\left(e v_{F} t\right)^{2} \mathcal{F}_{22} & 2\left(e v_{F} t\right)^{2} \mathcal{F}_{12} & 0 \\
0 & 2\left(e v_{F} t\right)^{2} \mathcal{F}_{12} & 2\left(e v_{F} t\right)^{2} \mathcal{F}_{11} & 0 \\
-2\left(e v_{F} t\right)^{2} \mathcal{F}_{12} & 0 & 0 & 0
\end{array}\right],
$$

and the eigenvalues are

$$
\begin{aligned}
& \alpha_{1}=\frac{1}{2}-e^{2} v_{F}^{2} t^{2}\left(\mathcal{F}_{11}+\mathcal{F}_{22}\right)+\frac{1}{2} \sqrt{4 e^{4} t^{4} v_{F}^{4}\left[\left(\mathcal{F}_{11}+\mathcal{F}_{22}\right)^{2}+4 \mathcal{F}_{12}^{2}\right]-4 e^{2} t^{2} v_{F}^{2}\left(\mathcal{F}_{11}+\mathcal{F}_{22}\right)+1} \\
& \alpha_{2}=\frac{1}{2}-e^{2} v_{F}^{2} t^{2}\left(\mathcal{F}_{11}+\mathcal{F}_{22}\right)-\frac{1}{2} \sqrt{4 e^{4} t^{4} v_{F}^{4}\left[\left(\mathcal{F}_{11}+\mathcal{F}_{22}\right)^{2}+4 \mathcal{F}_{12}^{2}\right]-4 e^{2} t^{2} v_{F}^{2}\left(\mathcal{F}_{11}+\mathcal{F}_{22}\right)+1} \\
& \alpha_{3}=e^{2} v_{F}^{2} t^{2}\left(\mathcal{F}_{11}+\mathcal{F}_{22}+\sqrt{\left(\mathcal{F}_{11}-\mathcal{F}_{22}\right)^{2}+4 \mathcal{F}_{12}^{2}}\right) \\
& \alpha_{4}=e^{2} v_{F}^{2} t^{2}\left(\mathcal{F}_{11}+\mathcal{F}_{22}-\sqrt{\left(\mathcal{F}_{11}-\mathcal{F}_{22}\right)^{2}+4 \mathcal{F}_{12}^{2}}\right)
\end{aligned}
$$

The first two eigenvalues cannot be negative because this would imply that $16 e^{4} t^{4} v_{F}^{4} \mathcal{F}_{12}^{2}<0$. The only eigenvalue that can be negative is $\alpha_{4}$. We shall therefore use the negativity as a measure of entanglement. The following expression is obtained for the negativity: 


$$
\mathcal{N}=e^{2} v_{F}^{2} t^{2}\left(\sqrt{\left(\mathcal{F}_{11}-\mathcal{F}_{22}\right)^{2}+4 \mathcal{F}_{12}^{2}}-\mathcal{F}_{11}-\mathcal{F}_{22}\right)
$$

The last equation is the sum of a local term $\mathcal{F}_{11}+\mathcal{F}_{22}$ that depends on the properties of just one of the graphene sheets and a nonlocal term $\sqrt{\left(\mathcal{F}_{11}-\mathcal{F}_{22}\right)^{2}+4 \mathcal{F}_{12}^{2}}$ that depends on the properties of both graphene sheets. This implies a direct competition between nonlocal, entangling exchange and local noise, which implies that in order to have entanglement between the graphene sheets the nonlocal term must overcome the single-graphene sheet noise excitations, as it occurs with atoms [2]. For the set of values of $d_{1} / L, d_{2} / L$ and $\Delta k=\left|\mathbf{k}_{\mathbf{2}}-\mathbf{k}_{\mathbf{2}}^{\prime}\right|$ in which $\mathcal{N}$ is positive, the double-layer graphene becomes entangled for times smaller than the light-crossing time $t<\frac{\left|d_{1}-d_{2}\right|}{c}$. In order to obtain analytical results in the case where $\Delta k=0$, instead of computing the sum over $n$ as done in Appendix A, we can compute the integral over $\Delta \mathbf{r}$. Then, $\mathcal{F}_{i j}$ can be written with the sum over $n$,

$$
\mathcal{F}_{i j}(\Delta k)=\frac{\gamma^{2}}{16 \pi^{2}} \sum_{n=1}^{\infty} \frac{\sin \left(\frac{n \pi d_{i}}{L}\right) \sin \left(\frac{n \pi d_{j}}{L}\right)}{\sqrt{\Delta k^{2}+\frac{n^{2} \pi^{2}}{L^{2}}}},
$$

which for the case where $\Delta k=0$ reads

$$
\begin{aligned}
\mathcal{F}_{i j}(k)= & \frac{L}{\pi} \ln \left[\frac{e^{-i\left(d_{i}+d_{j}\right) \frac{\pi}{L}}-1}{e^{i d_{i} \frac{\pi}{L}}-e^{i d_{j} \frac{\pi}{L}}}\right]+\frac{L^{3} \Delta k^{2}}{4 \pi^{3}}\left[L i_{3}\left(e^{-i\left(d_{1}-d_{2}\right) \frac{\pi}{L}}\right)\right. \\
& +L i_{3}\left(e^{i\left(d_{1}-d_{2}\right) \frac{\pi}{L}}\right)-L i_{3}\left(e^{-i\left(d_{1}+d_{2}\right) \frac{\pi}{L}}\right) \\
& \left.-L i_{3}\left(e^{i\left(d_{1}+d_{2}\right) \frac{\pi}{L}}\right)\right]+O\left(\Delta k^{4}\right)
\end{aligned}
$$

where we have expanded $\frac{1}{\sqrt{k^{2}+\frac{n^{2} n^{2}}{L^{2}}}} \sim \frac{L}{n \pi}-\frac{k^{2}}{2}\left(\frac{L}{n \pi}\right)^{3}+O\left(k^{4}\right)$. In

Figs. 3 and 4 the function $\mathcal{F}_{i j}$ and the negativity are shown as functions of the dimensionless parameters $d_{1} / L$ and $d_{2} / L$ for $\Delta \mathbf{k}=\mathbf{0}$. As expected, the negativity is larger

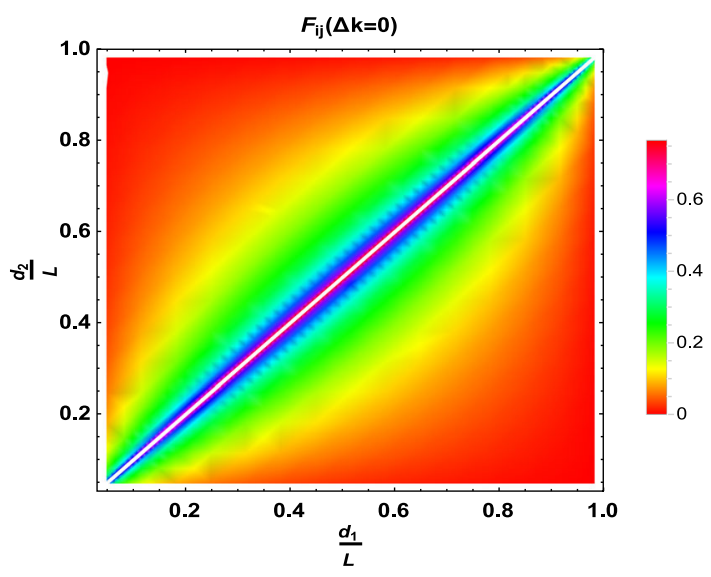

FIG. 3. The function $\mathcal{F}_{i j}$ as a function of the relative distance of both graphene sheets with respect to the cavity.

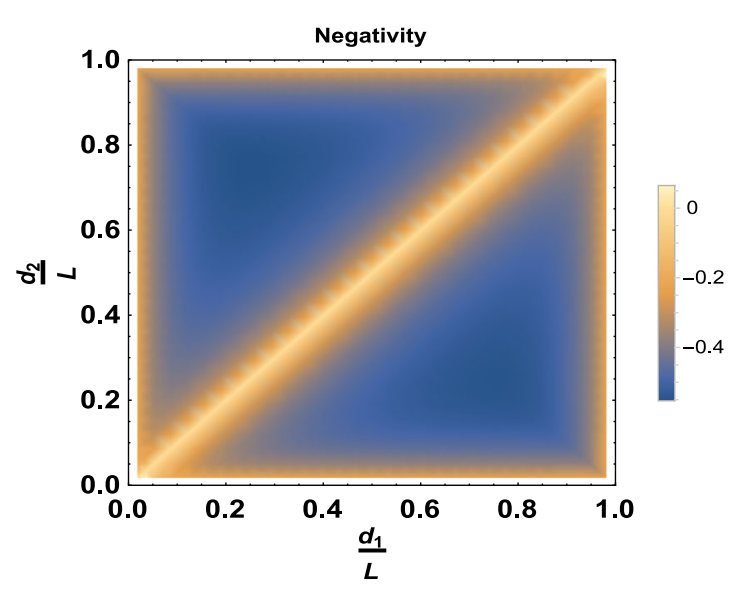

FIG. 4. The negativity measure as a function of each graphene layer's relative distance with respect the cavity.

when the layer separation is smaller at lowest order in $t$. An electron in one graphene layer has a nonzero probability of getting excited outside the light cone, but this probability is completely independent of the electron in the other graphene layer, so no information is being carried over a spacelike distance.

In turn, by numerically computing the integral in Eq. (18) for $\Delta t=0$ and $\Delta k \neq 0$ for different sets of values of $d_{1} / L$ and $d_{2} / L$, the negativity estimator shows a critical value of $\Delta k L \sim 3.2$ where the negativity changes sign (see Fig. 5). By considering $L=500 \mathrm{~nm}$ as a normal microcavity, the induced gap is $\epsilon_{G} \sim 6 \times 10^{-15} \mathrm{eV}$ which is smaller than typical induced gaps in normal semiconductors [47]. Following the same procedure, we can consider that the initial quantum state for the two electrons in each graphene sheet is given by eigenstates of the Hamiltonian which can be written as a superposition of the sublattice basis. This implies that the detector (which acts on the sublattice basis) will mix the eigenstates of the Hamiltonian. For simplicity, we can write the initial state as $\rho_{G}=|+,+\rangle\langle+,+|$ (see Fig. 6), and thus

$$
\left\langle s^{\prime}\left|\sigma_{\lambda}\right| s\right\rangle=\frac{1}{2}\left[s e^{i \theta}(1+\lambda)+s^{\prime} e^{-i \theta^{\prime}}(1-\lambda)\right],
$$

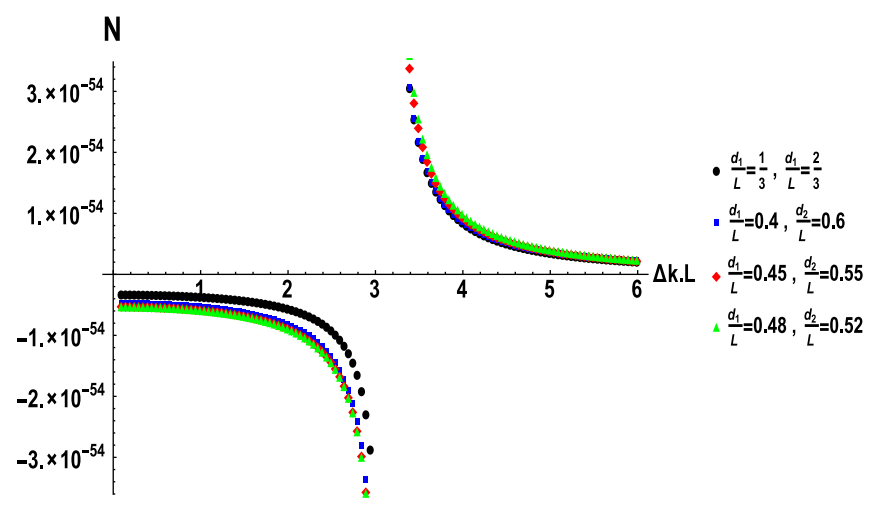

FIG. 5. The negativity as a function of the momentum transfer for different relative distances $d_{1} / L$ and $d_{2} / L$. 
where the basis $|s\rangle$ is $| \pm\rangle$. After a lengthy calculation, $\left\langle s_{1}^{\prime}, s_{2}^{\prime}\left|\left(\sigma_{-\lambda}^{(i)} \sigma_{-\lambda}^{(j)} \rho_{G}-2 \sigma_{-\lambda}^{(i)} \rho_{G} \sigma_{\lambda}^{(j)}+\rho_{G} \sigma_{\lambda}^{(i)} \sigma_{\lambda}^{(j)}\right)\right| s_{1}, s_{2}\right\rangle$ can be written as

$$
\left\langle s_{1}^{\prime}, s_{2}^{\prime}\left|\left(\sigma_{-\lambda}^{(i)} \sigma_{-\lambda}^{(j)} \rho_{G}-2 \sigma_{-\lambda}^{(i)} \rho_{G} \sigma_{\lambda}^{(j)}+\rho_{G} \sigma_{\lambda}^{(i)} \sigma_{\lambda}^{(j)}\right)\right| s_{1}, s_{2}\right\rangle=a_{11} \mathcal{F}_{11}+a_{22} \mathcal{F}_{22}+a_{12} \mathcal{F}_{12},
$$

where $a_{11}=-\delta_{1 s_{2}^{\prime}} \delta_{1 s_{2}} e^{i\left(\theta_{1}-\theta_{1}^{\prime}\right)}\left(1+s_{1} s_{1}^{\prime}\right), a_{22}=-\delta_{1 s_{1}} \delta_{1 s_{1}^{\prime}} e^{i\left(\theta_{1}-\theta_{1}^{\prime}\right)}\left(1+s_{2} s_{2}^{\prime}\right)$ and

$$
\begin{aligned}
a_{12}= & -e^{i\left(\theta_{1}-\theta_{2}^{\prime}\right)}\left(\delta_{1 s_{2}^{\prime}} \delta_{1 s_{1}}+\delta_{1 s_{1}^{\prime}} \delta_{1 s_{2}} s_{2^{\prime}} s_{1}\right)-e^{i\left(\theta_{2}-\theta_{1}^{\prime}\right)}\left(\delta_{1 s_{1}^{\prime}} \delta_{1 s_{2}}+\delta_{1 s_{2}^{\prime}} \delta_{1 s_{1}} s_{2} s_{1}^{\prime}\right) \\
& +e^{-i\left(\theta_{1}^{\prime}+\theta_{2}^{\prime}\right)}\left(\delta_{1 s_{1}^{\prime}} \delta_{1 s_{2}^{\prime}}+\delta_{1 s_{1}} \delta_{1 s 2} s_{1}^{\prime} s_{2}^{\prime}\right)+e^{i\left(\theta_{1}+\theta_{2}\right)}\left(\delta_{1 s_{1}} \delta_{1 s 2}+s_{1} s_{2} \delta_{1 s_{1}^{\prime}} \delta_{1 s_{2}^{\prime}}\right)
\end{aligned}
$$

where $\theta_{i}\left(\theta_{i}^{\prime}\right)$ is the initial (final) angle of the wave vector $\mathbf{k}_{\mathbf{i}}\left(\mathbf{k}_{\mathbf{i}}^{\prime}\right)$ that appears in the phase in Eq. (3). The normalized reduced quantum operator reads [see Eq. (8) in Ref. [15]]

$$
\rho=\left[\begin{array}{cccc}
1+\left(e v_{F} t\right)^{2}\left[A-D_{2}-D_{1}\right] & -\left(e v_{F} t\right)^{2} B_{+} & -\left(e v_{F} t\right)^{2} B_{-} & \left(e v_{F} t\right)^{2} C \\
\left(e v_{F} t\right)^{2} B_{+} & \left(e v_{F} t\right)^{2}\left[D_{2}-A\right] & \left(e v_{F} t\right)^{2} E & 0 \\
\left(e v_{F} t\right)^{2} B_{-} & \left(e v_{F} t\right)^{2} E & \left(e v_{F} t\right)^{2}\left[D_{1}-A\right] & 0 \\
\left(e v_{F} t\right)^{2} C & 0 & 0 & \left(e v_{F} t\right)^{2} A
\end{array}\right]
$$

where

$$
\begin{aligned}
A & =e^{-i\left(\theta_{1}^{\prime}+\theta_{2}^{\prime}\right)}\left[\mathcal{F}_{12}\left(-e^{i\left(\theta_{1}+\theta_{1}^{\prime}\right)}-e^{i\left(\theta_{2}+\theta_{2}^{\prime}\right)}+e^{i\left(\theta_{1}^{\prime}+\theta_{2}^{\prime}+\theta_{1}+\theta_{2}\right)}+1\right)-e^{i\left(\theta_{2}^{\prime}+\theta_{1}\right)}\left(\mathcal{F}_{11}+\mathcal{F}_{22}\right)\right] \\
B_{ \pm} & =\frac{1}{2} \mathcal{F}_{12} e^{-i\left(\theta_{1}^{\prime}+\theta_{2}^{\prime}\right)}\left(\mp 1+e^{i\left(\theta_{1}^{\prime}+\theta_{1}\right)}\right)\left( \pm 1+e^{i\left(\theta_{2}^{\prime}+\theta_{2}\right)}\right), \\
C & =\frac{1}{2} \mathcal{F}_{12}\left(e^{-i\left(\theta_{1}^{\prime}+\theta_{2}^{\prime}\right)}+e^{i\left(\theta_{1}+\theta_{2}\right)}\right), \\
D_{i} & =-\mathcal{F}_{i i} e^{-i\left(\theta_{1}^{\prime}-\theta_{1}\right)} \\
E & =-\frac{1}{2} \mathcal{F}_{12}\left(e^{i\left(\theta_{1}-\theta_{2}^{\prime}\right)}+e^{i\left(\theta_{2}-\theta_{1}^{\prime}\right)}\right) .
\end{aligned}
$$

From Eq. (30), the reduced operator is no longer an $X$ state due to the matrix elements $B_{ \pm}$; nevertheless, for specific choices of initial and final angles of the wave vectors, different kinds of entangled matrices can be obtained. For $B_{ \pm}=0$, the angles must obey $\theta_{1}^{\prime}+\theta_{1}=0$ and $\theta_{2}^{\prime}+\theta_{2}=0$ or $\theta_{1}^{\prime}+\theta_{1}=\pi$ and $\theta_{2}^{\prime}+\theta_{2}=\pi$. In the first case, $A$ does not depend on $\mathcal{F}_{12}$ and the matrix is identical to Eq. (20), but in the second case, $e^{i\left(\theta_{1}+\theta_{1}^{\prime}\right)}+$ $e^{i\left(\theta_{2}+\theta_{2}^{\prime}\right)}-e^{i\left(\theta_{1}^{\prime}+\theta_{2}^{\prime}+\theta_{1}+\theta_{2}\right)}-1$ does not vanish and $\mathcal{F}_{12}$ appears in the diagonal elements. In turn, when $\theta_{1}+\theta_{1}^{\prime}=\theta_{2}+\theta_{2}^{\prime}-\pi, E=0$ and the density matrix can

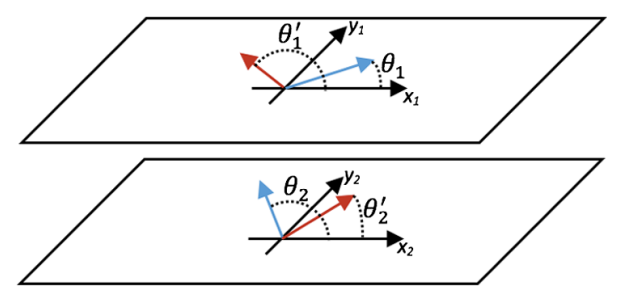

FIG. 6. Initial and final angles of both conduction electrons in each graphene sheet. no longer be related to all pure and mixed states by an entanglement-preserving unitary transformation such that the transformed state has the same entanglement as the input state, a property which is supported by strong numerical evidence [48]. The correlated angles at which the electrons in both layers scatter is related to the broken symmetry in double-layer graphene shown in Ref. [36]. The matrix-element dependence of $\rho$ with the initial and final angles implies that the nonlocal correlations are sensitive to the relative orientation of the electrons.

An operational two-party entanglement-harvesting protocol to detect this nonlocal correlation in double-layer graphene involves applying an external voltage on both layers, which can vary the carrier concentration in the material [49]. It is well known that graphene's density of states at the neutral point vanishes, which implies that there are no states to occupy and hence there are no carriers which could contribute to the electronic transport. An usual procedure to change the charge concentration is to use graphene as the second parallel plate of a capacitor, where the first plate is $\mathrm{SiO}_{2}$ and a back-gate voltage is applied 
perpendicular to the graphene sheet which creates an electrostatic potential drop between the sample and the gate electrode and shifts the Fermi level [50]. The distance between graphene layers should be an order of magnitude larger than the capacitor in order to not change the boundary conditions for the electromagnetic field used in the calculations [51]. By switching the back-gate voltage on and off in one graphene layer in the interval $\left[0, T_{1}\right]$ and performing the same procedure in the second layer in the interval $\left[T_{2}, T_{2}^{\prime}\right]$ (where $T_{2}^{\prime}-T_{2}=T_{1}$ and the initial time at which the second back-gate voltage is turned on obeys $T_{2}<\frac{d_{1}-d_{2}}{c}$ ) and by measuring the current in each graphene layer [52], it is possible to detect nonlocal correlation even if both electrons do not exchange real photons. ${ }^{5}$ An improvement to the setup is to introduce a dielectric in the whole cavity that changes the refractive index and the velocity of light in order to decrease the time intervals at which the back-gate voltages are switched on and off [51].

Summing up, we have presented a new physical effect of vacuum fluctuations which is associated with quantum nonlocality in double-layer graphene, which allows to study relativistic quantum effects in the laboratory. It should be stressed that this effect stands in contrast to other vacuum phenomena, such as the Lamb shift or the Casimir effect [54], which to some extent can be emulated by classical stochastic local noise.

\section{CONCLUSIONS}

In this work we have performed a detailed study of the phenomenon of entanglement harvesting from the vacuum state of the electromagnetic field in double-layer graphene for different initial states for the electrons. By considering that each graphene sheet interacts with the vacuum electromagnetic field state and by partially tracing the degrees of freedom of this field, the reduced quantum state of the electrons in different layers gets entangled for times smaller than the time of flight of light between the sheets. By using time-dependent perturbation theory up to second order, the negativity measure of entanglement has been computed. We have exhaustively analyzed the case in which both electrons are in one of the pseudospin states, showing that for time scales smaller than the light-crossing time between both layers, both electrons are correlated due to the tails of the virtual photon propagator. In turn, we have shown that when both electrons are in the conduction band, the reduced density matrix reduces to an $X$ state for $\theta_{1}^{\prime}+\theta_{1}=0$ and $\theta_{2}^{\prime}+\theta_{2}=0$ or $\theta_{1}^{\prime}+\theta_{1}=\pi$ and $\theta_{2}^{\prime}+\theta_{2}=\pi$, and for general angles the bipartite quantum

\footnotetext{
${ }^{5}$ The two voltages are switched on for the same amount of time but with a time delay between them, which implies that the worldsheet of the second graphene layer lies outside the light cone of the worldsheet of the first graphene layer (see Fig. 1 of Ref. [53]).
}

state becomes highly entangled with broken electron-hole symmetry.

\section{ACKNOWLEDGMENTS}

J.S. A. would like to thank F. Guinea for fruitful discussions. This paper was partially supported by grants from CONICET (Argentina National Research Council) and Universidad Nacional del Sur (UNS), by ANPCyT (Agencia Nacional de Promoción Científica y Tecnológica) through PICT 2014-1351, and by PIP-CONICET research Grants No. 114-200901-00272 and No. 114-200901-00068. J. S. A. is member of CONICET.

\section{APPENDIX A: PHOTON PROPAGATOR}

In order to compute the photon propagator of Eq. (12),

$$
\begin{aligned}
& \left\langle\Omega\left|T A_{\lambda}^{(i)}\left(\mathbf{r}_{\mathbf{1}}, t_{1}\right) A_{\lambda^{\prime}}^{(j)}\left(\mathbf{r}_{2}, t_{2}\right)\right| \Omega\right\rangle \\
& =\delta_{\lambda \lambda^{\prime}} \sum_{n=1}^{\infty} \gamma^{2} \sin \left(\frac{\pi n d_{i}}{L}\right) \sin \left(\frac{\pi n d_{j}}{L}\right) \\
& \quad \times \int \frac{d^{2} \mathbf{q}}{(2 \pi)^{2}} \frac{e^{i \mathbf{q}\left(\mathbf{r}_{1}-\mathbf{r}_{2}\right)} e^{i \omega_{\mathbf{n}, \mathbf{q}}\left(\mathbf{t}_{2}-\mathbf{t}_{1}\right)}}{\omega_{n, \mathbf{q}}},
\end{aligned}
$$

we can apply the Schwinger time representation procedure by introducing a new variable of integration $q_{0}$,

$$
\frac{e^{i \omega_{\mathbf{n}, \mathbf{q}}\left(\mathbf{t}_{2}-\mathbf{t}_{1}\right)}}{\omega_{n, \mathbf{q}}}=\int_{-\infty}^{\infty} \frac{d q_{0}}{2 \pi i} \frac{2 e^{i q_{0}\left(\mathbf{t}_{2}-\mathbf{t}_{1}\right)}}{q_{0}^{2}-q^{2}-\left(\frac{n \pi}{L}\right)^{2}} .
$$

The $q_{0}$ integration can be computed using the residue theorem, and the contour contains the $q_{0}$ real line and the semicircle of radius $R$, where $R \rightarrow \infty$ and where the contour encloses the pole located at $q_{0}=\omega_{n, \mathbf{q}}=$ $\sqrt{q^{2}+\left(\frac{n \pi}{L}\right)^{2}}$. Then, we can apply the Wick rotation to the Euclidean space by defining $q_{0}=i p_{0}$ and $\mathbf{q}=\mathbf{p}$; thus, $d^{2} \mathbf{q} \mathbf{d} \mathbf{q}_{\mathbf{0}}=\mathbf{i \mathbf { d } ^ { 3 } \mathbf { p }}$ and $q_{0}^{2}-\mathbf{q} \cdot \mathbf{q}=-p_{0}^{2}-\mathbf{p}^{\mathbf{2}}=-p^{2}$ and Eq. (A1) becomes

$$
\begin{aligned}
& \int \frac{d^{2} \mathbf{q}}{(2 \pi)^{2}} \int_{-\infty}^{\infty} \frac{d q_{0}}{2 \pi i} \frac{2 e^{i \mathbf{q}\left(\mathbf{r}_{1}-\mathbf{r}_{2}\right)} e^{-i q_{0}\left(\mathbf{t}_{1}-\mathbf{t}_{2}\right)}}{q_{0}^{2}-q^{2}-\left(\frac{n \pi}{L}\right)^{2}} \\
& \rightarrow-\int \frac{d^{3} \mathbf{p}}{(2 \pi)^{3}} \frac{2 e^{i p \cdot x}}{p^{2}+\left(\frac{n \pi}{L}\right)^{2}},
\end{aligned}
$$

where $x=(\Delta t,-\Delta \mathbf{r})$. The last integral can be computed by considering spherical coordinates $d^{3} \mathbf{p}=$ $p^{2} d p \sin \theta_{p} d \theta_{p} d \phi_{p}$ and by writing $p \cdot x=p|x| \cos \theta_{p}$, where $\theta_{p}$ is the angle between the momentum $\mathbf{p}$ and the vector $x,|x|=\sqrt{c^{2} \Delta t^{2}-|\Delta \mathbf{r}|^{2}}$, and $\Delta \mathbf{r}=\mathbf{r}_{1}-\mathbf{r}_{2}$. Computing the integrals over $\theta_{p}$ and $\phi_{p}$, we obtain 


$$
\Delta_{\lambda}^{(i, j)}(\Delta t, \Delta \mathbf{r})=-\frac{\delta_{\lambda \lambda^{\prime}}}{\pi^{2}|x|} \sum_{n=1}^{\infty} \gamma^{2} \sin \left(\frac{\pi n d_{i}}{L}\right) \sin \left(\frac{\pi n d_{j}}{L}\right) \int_{0}^{\infty} \frac{p d p \sin (p|x|)}{p^{2}+\left(\frac{n \pi}{L}\right)^{2}}
$$

Finally, the integral over $p$ reads

$$
\int_{0}^{\infty} \frac{p d p \sin (p|x|)}{p^{2}+\left(\frac{n \pi}{L}\right)^{2}}=-i \theta\left(x^{2}\right) \frac{1}{4 \pi\left(x^{2}-i \epsilon\right)^{\frac{1}{2}}} e^{-i \frac{n \pi}{L} \sqrt{x^{2}-i \epsilon}}+\theta\left(-x^{2}\right) \frac{1}{4 \pi\left(-x^{2}+i \epsilon\right)^{\frac{1}{2}}} e^{-\frac{n \pi}{L} \sqrt{-x^{2}+i \epsilon}},
$$

where we have used Eq. (27) of Ref. [55], and the sum over $n$ reads

$$
\begin{aligned}
& \Delta_{\lambda}^{(i, j)}(|x|)=-\delta_{\lambda \lambda^{\prime}} \frac{\gamma^{2}}{2 \pi|x|} \sum_{n=1}^{\infty} \sin \left(\frac{n \pi d_{i}}{L}\right) \sin \left(\frac{n \pi d_{j}}{L}\right) e^{-n \pi \frac{|x|}{L}}= \\
& \Delta_{\lambda}^{(i, j)}(|x|)=-\frac{\delta_{\lambda \lambda^{\prime}}}{16 \pi|x|} \frac{\gamma^{2} \sin \left(\frac{\pi d_{i}}{L}\right) \sin \left(\frac{\pi d_{j}}{L}\right) \sinh \left(\frac{\pi|x|}{L}\right)}{\sin \left(\frac{\pi\left(d_{i}-d_{j}-i|x|\right)}{2 L}\right) \sin \left(\frac{\pi\left(d_{i}+d_{j}-i|x|\right)}{2 L}\right) \sin \left(\frac{\pi\left(d_{i}-d_{j}+i|x|\right)}{2 L}\right) \sin \left(\frac{\pi\left(d_{i}+d_{j}+i|x|\right)}{2 L}\right)},
\end{aligned}
$$

which is the desired result for the photon propagator in the planar microcavity.

\section{APPENDIX B: SECOND ORDER CONTRIBUTION TO THE REDUCED QUANTUM OPERATOR}

In order to obtain Eq. (16) we must compute the matrix elements of the reduced density matrix $\rho(t)=\operatorname{Tr}_{\phi}\left(U^{(2)} \rho_{0}+U^{(1)} \rho U^{(1) \dagger}+\rho_{0} U^{(2) \dagger}\right)$, that is, $\left\langle\mathbf{k}_{\mathbf{1}}^{\prime}, s_{1}^{\prime}, \mathbf{k}_{\mathbf{2}}^{\prime}, s_{2}^{\prime}|\rho(t)| \mathbf{k}_{\mathbf{1}}, s_{1}, \mathbf{k}_{\mathbf{2}}, s_{2}\right\rangle$, where $\mathbf{k}_{\mathbf{i}}^{\prime}, s_{i}^{\prime}$ are the labels for the wave vector and $s= \pm 1$ is the band index. It should be noted that these matrix elements do not depend on the photon quantum states due to the partial trace over these degrees of freedom. For simplicity, we will compute the matrix elements of the first term of $\rho(t)$, that is, $\left\langle\mathbf{k}_{\mathbf{1}}^{\prime}, s_{1}^{\prime}, \mathbf{k}_{\mathbf{2}}^{\prime}, s_{2}^{\prime}\left|\operatorname{Tr}_{\phi}\left(U^{(2)} \rho_{0}\right)\right| \mathbf{k}_{\mathbf{1}}, s_{1}, \mathbf{k}_{\mathbf{2}}, s_{2}\right\rangle$. We can write $\operatorname{Tr}_{\phi}\left(U^{(2)} \rho_{0}\right)$ as

$$
\operatorname{Tr}_{\phi}\left(U^{(2)} \rho_{0}\right)=-\left(e v_{F}\right)^{2} \sum_{i, j=1,2 ; \lambda, \lambda^{\prime}} \int_{0}^{t} \int_{0}^{t} d t_{1} d t_{2} \times \operatorname{Tr}_{\phi}\left[e^{i\left(H_{0}+H_{F}\right) t_{1}} \sigma_{-\lambda}^{(i)} A_{\lambda}^{(i)} e^{-i\left(H_{0}+H_{F}\right) t_{1}} e^{i\left(H_{0}+H_{F}\right) t_{2}} \sigma_{-\lambda^{\prime}}^{(j)} A_{\lambda^{\prime}}^{(j)} e^{-i\left(H_{0}+H_{F}\right) t_{2}} \rho_{G}\right],
$$

with $\rho_{0}=|\Omega\rangle\langle\Omega| \rho_{G}$, where $\rho_{G}$ is the initial density operator of the two-electron system, $\rho_{G}=\left|\mathbf{k}_{\mathbf{1}}^{(\mathbf{0})}, s_{1}^{(0)}, \mathbf{k}_{\mathbf{2}}^{(\mathbf{0})}, s_{2}^{(0)}\right\rangle$ $\left\langle\mathbf{k}_{\mathbf{1}}^{(\mathbf{0})}, s_{1}^{(0)}, \mathbf{k}_{\mathbf{2}}^{(\mathbf{0})}, s_{2}^{(0)}\right|$, where $\mathbf{k}_{\mathbf{i}}^{(\mathbf{0})}$ and $s_{i}^{(0)}$ are the initial wave vectors and valence/conduction (or sublattice) indices. The last equation can be written as

$$
\operatorname{Tr}_{\phi}\left(U^{(2)} \rho_{0}\right)=-\left(e v_{F}\right)^{2} \sum_{i, j=1,2 ; \lambda, \lambda^{\prime}} \int_{0}^{t} \int_{0}^{t} d t_{1} d t_{2}\left\langle\Omega\left|A_{\lambda}^{(i)}\left(t_{1}\right) A_{\lambda^{\prime}}^{(j)}\left(t_{2}\right)\right| \Omega\right\rangle \sigma_{-\lambda}^{(i)}\left(t_{1}\right) \sigma_{-\lambda^{\prime}}^{(j)}\left(t_{2}\right) \rho_{G},
$$

where $\sigma_{-\lambda}^{(i)}\left(t_{1}\right)=e^{i H_{0} t_{1}} \sigma_{-\lambda}^{(i)} e^{-i H_{0} t_{1}}, \sigma_{-\lambda^{\prime}}^{(j)}\left(t_{2}\right)=e^{i H_{0} t_{2}} \sigma_{-\lambda^{\prime}}^{(j)} e^{-i H_{0} t_{2}}, A_{\lambda}^{(i)}\left(t_{1}\right)=e^{i H_{F} t_{1}} A_{\lambda}^{(i)} e^{-i H_{F} t_{1}}$, and $A_{\lambda^{\prime}}^{(j)}\left(t_{2}\right)=e^{i H_{F} t_{2}} A_{\lambda^{\prime}}^{(j)} e^{-i H_{F} t_{2}}$. We then apply $\left\langle\mathbf{k}_{\mathbf{1}}^{\prime}, s_{1}^{\prime}, \mathbf{k}_{\mathbf{2}}^{\prime}, s_{2}^{\prime}\right|$ and $\left|\mathbf{k}_{\mathbf{1}}, s_{1}, \mathbf{k}_{\mathbf{2}}, s_{2}\right\rangle$ in the coordinate representation,

$$
\begin{aligned}
\left\langle\mathbf{k}_{\mathbf{1}}^{\prime}, s_{1}^{\prime}, \mathbf{k}_{2}^{\prime}, s_{2}^{\prime}\left|\operatorname{Tr}_{\phi}\left(U^{(2)} \rho_{0}\right)\right| \mathbf{k}_{\mathbf{1}}, s_{1}, \mathbf{k}_{\mathbf{2}}, s_{2}\right\rangle= & -\left(e v_{F}\right)^{2} \sum_{i, j=1,2 ; \lambda, \lambda^{\prime}} \int d^{2} \mathbf{r}_{\mathbf{1}} \int d^{2} \mathbf{r}_{\mathbf{2}} \int_{0}^{t} \int_{0}^{t} d t_{1} d t_{2} e^{i \mathbf{k}_{1} \cdot \mathbf{r}_{1}} e^{i \mathbf{k}_{2} \cdot \mathbf{r}_{2}} e^{-i \mathbf{k}_{1}^{\prime} \cdot \mathbf{r}_{1}} e^{-i \mathbf{k}_{2}^{\prime} \cdot \mathbf{r}_{2}} \\
& \times\left\langle\Omega\left|A_{\lambda}^{(i)}\left(\mathbf{r}_{1}, d_{i}, t_{1}\right) A_{\lambda^{\prime}}^{(j)}\left(\mathbf{r}_{2}, d_{j}, t_{2}\right)\right| \Omega\right\rangle\left\langle s_{1}^{\prime}, s_{2}^{\prime}\left|\sigma_{-\lambda}^{(i)}\left(t_{1}\right) \sigma_{-\lambda^{\prime}}^{(j)}\left(t_{2}\right) \rho_{G}\right| s_{1}, s_{2}\right\rangle .
\end{aligned}
$$

In Appendix A it was shown that $\left\langle\Omega\left|T A_{\lambda}^{(i)}\left(\mathbf{r}_{1}, t_{1}\right) A_{\lambda^{\prime}}^{(j)}\left(\mathbf{r}_{2}, t_{2}\right)\right| \Omega\right\rangle=\Delta_{\lambda}^{(i, j)}(|x|)=\delta_{\lambda \lambda^{\prime}} F_{i j}(|x|)$, where $|x|=\sqrt{\Delta t^{2}-|\Delta \mathbf{r}|^{2}}$. Then,

$$
\begin{aligned}
& \left\langle\mathbf{k}_{\mathbf{1}}^{\prime}, s_{1}^{\prime}, \mathbf{k}_{\mathbf{2}}^{\prime}, s_{2}^{\prime}\left|\operatorname{Tr}_{\phi}\left(U^{(2)} \rho_{0}\right)\right| \mathbf{k}_{\mathbf{1}}, s_{1}, \mathbf{k}_{\mathbf{2}}, s_{2}\right\rangle \\
& \quad=-\left(e v_{F}\right)^{2} \sum_{i, j=1,2 ; \lambda} \int_{0}^{t} \int_{0}^{t} \int d^{2} \mathbf{r}_{\mathbf{1}} \int d^{2} \mathbf{r}_{2} d t_{1} d t_{2} e^{i\left(\mathbf{k}_{1}-\mathbf{k}_{1}^{\prime}\right) \cdot \mathbf{r}_{1}} e^{i\left(\mathbf{k}_{2}-\mathbf{k}_{2}^{\prime}\right) \cdot \mathbf{r}_{2}} F_{i j}(|x|)\left\langle s_{1}^{\prime}, s_{2}^{\prime}\left|\sigma_{-\lambda}^{(i)}\left(t_{1}\right) \sigma_{-\lambda}^{(j)}\left(t_{2}\right) \rho_{G}\right| s_{1}, s_{2}\right\rangle .
\end{aligned}
$$


By performing the change of variables $\Delta r=\mathbf{r}_{1}-\mathbf{r}_{2}$, we have

$$
\begin{aligned}
\left\langle\mathbf{k}_{\mathbf{1}}^{\prime}, s_{1}^{\prime}, \mathbf{k}_{\mathbf{2}}^{\prime}, s_{2}^{\prime}\left|\operatorname{Tr}_{\phi}\left(U^{(2)} \rho_{0}\right)\right| \mathbf{k}_{\mathbf{1}}, s_{1}, \mathbf{k}_{\mathbf{2}}, s_{2}\right\rangle= & -\left(e v_{F}\right)^{2} \sum_{i, j=1,2 ; \lambda} \int_{0}^{t} \int_{0}^{t} d t_{1} d t_{2} \int d^{2} \mathbf{r}_{\mathbf{1}} \int d^{2} \mathbf{r}_{2} e^{i\left(\mathbf{k}_{1}-\mathbf{k}_{1}^{\prime}+\mathbf{k}_{2}-\mathbf{k}_{2}^{\prime}\right) \cdot \mathbf{r}_{1}} e^{-i\left(\mathbf{k}_{2}-\mathbf{k}_{2}^{\prime}\right) \cdot \Delta \mathbf{r}} \\
& \times F_{i j}\left(\sqrt{\Delta t^{2}-|\Delta \mathbf{r}|^{2}}\right)\left\langle s_{1}^{\prime}, s_{2}^{\prime}\left|\sigma_{-\lambda}^{(i)}\left(t_{1}\right) \sigma_{-\lambda}^{(j)}\left(t_{2}\right) \rho_{G}\right| s_{1}, s_{2}\right\rangle .
\end{aligned}
$$

Integrating over $\mathbf{r}_{1}$, we have

$$
\begin{aligned}
& \left\langle\mathbf{k}_{\mathbf{1}}^{\prime}, s_{1}^{\prime}, \mathbf{k}_{\mathbf{2}}^{\prime}, s_{2}^{\prime}\left|\operatorname{Tr}_{\phi}\left(U^{(2)} \rho_{0}\right)\right| \mathbf{k}_{\mathbf{1}}, s_{1}, \mathbf{k}_{\mathbf{2}}, s_{2}\right\rangle \\
& \quad=-\left(e v_{F}\right)^{2} \delta\left(\mathbf{k}_{\mathbf{1}}-\mathbf{k}_{\mathbf{1}}^{\prime}+\mathbf{k}_{\mathbf{2}}-\mathbf{k}_{\mathbf{2}}^{\prime}\right) \sum_{i, j=1,2 ; \lambda} \int_{0}^{t} \int_{0}^{t} d t_{1} d t_{2}\left\langle s_{1}^{\prime}, s_{2}^{\prime}\left|\sigma_{-\lambda}^{(i)}\left(t_{1}\right) \sigma_{-\lambda}^{(j)}\left(t_{2}\right) \rho_{G}\right| s_{1}, s_{2}\right\rangle \mathcal{F}_{i j}\left(\mathbf{k}_{\mathbf{2}}-\mathbf{k}_{\mathbf{2}}^{\prime}\right),
\end{aligned}
$$

where $\mathcal{F}_{i j}\left(\mathbf{k}_{\mathbf{2}}-\mathbf{k}_{\mathbf{2}}^{\prime}\right)$ is the Fourier transform of $F_{i j}\left(\sqrt{\Delta t^{2}-|\Delta \mathbf{r}|^{2}}\right)$,

$$
\mathcal{F}_{i j}\left(\mathbf{k}_{\mathbf{2}}-\mathbf{k}_{\mathbf{2}}^{\prime}, \Delta t\right)=\int d^{2} \Delta \mathbf{r} e^{-i\left(\mathbf{k}_{2}-\mathbf{k}_{2}^{\prime}\right) \cdot \Delta \mathbf{r}} F_{i j}\left(\sqrt{\Delta t^{2}-|\Delta \mathbf{r}|^{2}}\right)
$$

An identical procedure can be applied to $\left\langle\mathbf{k}_{\mathbf{1}}^{\prime}, s_{1}^{\prime}, \mathbf{k}_{\mathbf{2}}^{\prime}, s_{2}^{\prime}\left|\operatorname{Tr}_{\phi}\left(U^{(1)} \rho_{0} U^{(1) \dagger}\right)\right| \mathbf{k}_{\mathbf{1}}, s_{1}, \mathbf{k}_{\mathbf{2}}, s_{2}\right\rangle$ and $\left\langle\mathbf{k}_{\mathbf{1}}^{\prime}, s_{1}^{\prime}, \mathbf{k}_{\mathbf{2}}^{\prime}, s_{2}^{\prime}\right| \operatorname{Tr}_{\phi}\left(\rho_{0} U^{(2) \dagger}\right)$ $\left|\mathbf{k}_{\mathbf{1}}, s_{1}, \mathbf{k}_{\mathbf{2}}, s_{2}\right\rangle$, and the results are shown in Eqs. (14) and (15).

[1] A. Valentini, Phys. Lett. A 153, 321 (1991).

[2] B. Reznik, A. Retzker, and J. Silman, Phys. Rev. A 71, 042104 (2005).

[3] A. Pozas-Kerstjens and E. Martin-Martinez, Phys. Rev. D 92, 064042 (2015).

[4] R. H. Jonsson, E. Martin-Martinez, and A. Kempf, Phys. Rev. Lett. 114, 110505 (2015).

[5] S. W. Hawking, M. J. Perry, and A. Strominger, Phys. Rev. Lett. 116, 231301 (2016).

[6] A. Almheiri, D. Marolf, J. Polchinski, and J. Sully, J. High Energy Phys. 02 (2013) 62.

[7] M. Hotta, Phys. Rev. D 78, 045006 (2008).

[8] J. S. Ardenghi, Phys. Rev. D 91, 085006 (2015).

[9] J. S. Ardenghi, Int. J. Mod. Phys. A 33, 1850081 (2018).

[10] B. Reznik, Found. Phys. 33, 167 (2003).

[11] E. Martin-Martinez, E. G. Brown, W. Donnelly, and A. Kempf, Phys. Rev. A 88, 052310 (2013).

[12] G. Salton, R. B. Mann, and N. C. Menicucci, New J. Phys. 17, 035001 (2015).

[13] G. V. Steeg and N. C. Menicucci, Phys. Rev. D 79, 044027 (2009).

[14] E. Martin-Martinez and N. C. Menicucci, Classical Quantum Gravity 29, 224003 (2012).

[15] E. Martin-Martinez, A. R. H. Smith, and D. R. Terno, Phys. Rev. D 93, 044001 (2016).

[16] B. S. DeWitt, S. W. Hawking, and W. Israel, General Relativity: An Einstein Centenary Survey (Cambridge University Press, Cambridge, England, 1979).

[17] S. J. Olson and T. C. Ralph, Phys. Rev. Lett. 106, 110404 (2011).
[18] S. J. Olson and T.C. Ralph, Phys. Rev. A 85, 012306 (2012).

[19] C. Sabin, B. Peropadre, M. del Rey, and E. Martin-Martinez, Phys. Rev. Lett. 109, 033602 (2012).

[20] A. Pozas-Kerstjens and E. Martin-Martinez, Phys. Rev. D 94, 064074 (2016).

[21] M. O. Scully and M. S. Zubairy, Quantum Optics (Cambridge University Press, Cambridge, England, 1997).

[22] E. Martin-Martinez, Phys. Rev. D 92, 104019 (2015).

[23] A. Geim, Science 324, 1530 (2009).

[24] F. Escudero, J. S. Ardenghi, L. Sourrouille, and P. Jasen, J. Magn. Magn. Mater. 429, 294 (2017).

[25] F. Escudero, J. S. Ardenghi, and P. Jasen, J. Phys. Condens. Matter 30, 275803 (2018).

[26] S. Das Sarma, S. Adam, E. H. Hwang, and E. Rossi, Rev. Mod. Phys. 83, 407 (2011).

[27] K. S. Novoselov, A. K. Geim, S. V. Morozov, D. Jiang, M. I. Katsnelson, I. V. Grigorieva, S. V. Dubonos, and A. A. Firsov, Nature (London) 438, 197 (2005).

[28] A. H. Castro Neto, F. Guinea, N. M. R. Peres, K. S. Novoselov, and A. K. Geim, Rev. Mod. Phys. 81, 109 (2009).

[29] C. W. J. Beenakker, Rev. Mod. Phys. 80, 1337 (2008).

[30] I. V. Fialkovsky, V. N. Marachevsky, and D. V. Vassilevich, Phys. Rev. B 84, 035446 (2011).

[31] O. V. Kibis, Phys. Rev. B 81, 165433 (2010).

[32] T. C. H. Liew, I. A. Shelykh, and G. Malpuech, Physica (Amsterdam) 43E, 1543 (2011).

[33] T. Low, A. Chaves, J. D. Caldwell, A. Kumar, N. X. Fang, P. Avouris, T. F. Heinz, F. Guinea, L. Martin-Moreno, and F. Koppensm, Nat. Mater. 16, 182 (2017). 
[34] V. B. Berestetskii, E. M. Lifshitz, and L. P. Pitaevskii, Quantum Electrodynamics (Pergamon, New York, 1982).

[35] T Stauber and G. Gomez-Santos, New J. Phys. 14, 105018 (2012).

[36] R. V. Gorbachev, A. K. Geim, M. I. Katsnelson, K. S. Novoselov, T. Tudorovskiy, I. V. Grigorieva, A. H. MacDonald, S. V. Morozov, K. Watanabe, T. Taniguchi, and L. A. Ponomarenko, Nat. Phys. 8, 896 (2012).

[37] O. V. Kibis, O. Kyriienko, and I. A. Shelykh, Phys. Rev. B 87, 245437 (2013).

[38] S. Schlicht, Classical Quantum Gravity 21, 4647 (2004).

[39] E. Martın-Martınez, M. Montero, and M. del Rey, Phys. Rev. D 87, 064038 (2013).

[40] M. Ali, A. R. P. Rau, and G. Alber, Phys. Rev. A 81, 042105 (2010).

[41] T. Yu and J. H. Eberly, Quantum Inf. Comput. 7, 459 (2007).

[42] R. F. Werner, Phys. Rev. A 40, 4277 (1989).

[43] P. E. M. F. Mendonca, M. A. Marchiolli, and D. Galetti, Ann. Phys. (Amsterdam) 351, 79 (2014).

[44] A. Peres, Phys. Rev. Lett. 77, 1413 (1996).

[45] M. Horodecki, P. Horodecki, and R. Horodecki, Phys. Lett. A 223, 1 (1996).
[46] G. Vidal and R.F. Werner, Phys. Rev. A 65, 032314 (2002).

[47] O. V. Kibis, K. B. Arnardottir, and I. A. Shelykh, Phys. Rev. A 90, 055802 (2014).

[48] S. R. Hedemann, arXiv:1310.7038.

[49] M. F. Craciun, S. Russo, M. Yamamoto, and S. Tarucha, Nano Today 6, 42 (2011).

[50] A. Das, B. Chakraborty, and K. Sood, Graphene and Its Fascinating Attributes (World Scientific, Singapore, 2011), Chap. 7.

[51] S. M. Badalyan and F. M. Peeters, Phys. Rev. B 85, 195444 (2012).

[52] K. Tsukagoshi, H. Miyazaki, S.-L. Li, A. Kumatani, H. Hiura, and A. Kanda, Graphene and its Fascinating Attributes (World Scientific, Singapore, 2011), p. 179.

[53] E. Martın-Martınez and B. C. Sanders, New J. Phys. 18, 043031 (2016).

[54] M. B. Farias, C. D. Fosco, F. C. Lombardo, and F. D. Mazzitelli, Phys. Rev. D 95, 065012 (2017).

[55] H. Zhang, K. Feng, S. Qiu, A. Zhao, and X. Li, Chin. Phys. C 34, 1576 (2010). 